\title{
The Path of UNITY or the Path of UNREAL? A Comparative Study on Suitability for Game Development
}

\author{
Hussain Ali Juma Al Lawati MIDDLE EAST COLLEGE
}

Game development has become one of the major sectors in the world of programming. However, it requires a serious and a huge understanding on the features and usability with respect to different features of a game. Here the research intends give a detailed comparison between Unity and Unreal Engine that assists beginner game developers to choose the right engine for their development. The research is supported by literature reviews on Unity development and management as well as Unreal Engine's development kit evolution. Each of the engines require the application of certain programming. Pros and Cons for both engines are mentioned with aids from several articles and reviewers' opinions. In addition, visuals are attached to show the exact differences between both engines. This comparative research shall act as a guide to the game developers to choose wisely based on the requirements in terms of performance, functionality, configurability, practicability and accessibility. The results of this comparison will open doors of knowledge for us about the world of game development engines and unclear the fog for all game development seekers.

\section{Introduction}

The world is growing fast, and industries are expanding enormously and rapidly. Game development is one of those industries that has reached the a level where it keeps on improving non-stop regularly. However, even if it is that a big of an industry, there are many people who are semi-blind towards what game development really is. Game development is an art like any other art which aims at creating games and describing the design, development and output of the game. Developing a game can be done by a game development studio or by an individually, it only what really matters if is the interactivity between the players and the content and its effect in the game's element. Even with this industry's popularity, many people still think that game development can only be done by programmers, it is a very common false thought which is absolutely false about it. Game development is an industry that consists of several roles, of not only programmers, but designers, sound designers, artists and many others roles. If you want to get involved in the game development process, you do not really need to write a single line of code. Example: An artist can design assets for you, meanwhile the developer works on programming the object's movements. (FreeCodeCamp, n.d.).

To make games creating easier, people nowadays tend to use game engines to develop new games. Game Engines are software frameworks that allowthe users to build and create games in various platforms (game consoles, mobiles and Personal computers). Generally, it provides features like graphics rendering, collision detection, memory management and many other features. Some Game Engines are very difficult to learn, like Unreal Engine and CryEngine. On the other hand, we have the Unity Game Engine, which is considered as a moderate-level of difficulty, and hence it is a beginner friendly Game Engine (FreeCodeCamp, n.d.). Here is the list of some other Game Engines: Game Maker, Constructor 2, Constructor 3, Twine and Frostbite.

The ultimate objective is to provide support and guidance to choose the suitable game that will be based on the developer's needs and project type as well as how experienced is the developer is. 


\section{Methodology}

Game engines has have components, and (as stated before), they are frameworks. To display these components in an understandable way, a structure was adopted which emphasizes those components. In addition, literature from different reliable resources also assisted the research and confirms the gathered information. A part of a detailed table was used to show the full information of the game engines features and the differences between them, all the articles read and website referred to have been listed below in the List of Reference

\section{Game Engines}

- Unity Engine: Unity is one of the most famous game engines around the world. It uses C\# and UnityScript as scripting language (Petty, J. n.d.). In addition, Unity can be used for game development without using codes, in such cases Assets can be used to do so (Asset Example: PlayMaker). Unity projects can be a done by a solo developer or in teams. To add more to that, it is much easier to work on prototypes in Unity and less complicated..... the sentence is not complete.

- Unreal Engine: Unreal Engine is considered the highest and most competitive game engine that rivals Unity. Unreal Engine uses $\mathrm{C}++$ language to code scripts that will run the game. Unreal Engine also uses blueprints, blueprints are visual scripts that allows user to prototype the game in an easy way without typing a single code (Denham, T. n.d).. Unreal Engine is more difficult to use than Unity, however it provides better graphics quality.

\section{Game Engines Components}

The Analysis of different literature on game development proves that all game engines have 5 main components (Šmíd A. 2017) in them and each component has its own functionalities and features, the diagram below demonstrates as follows: 


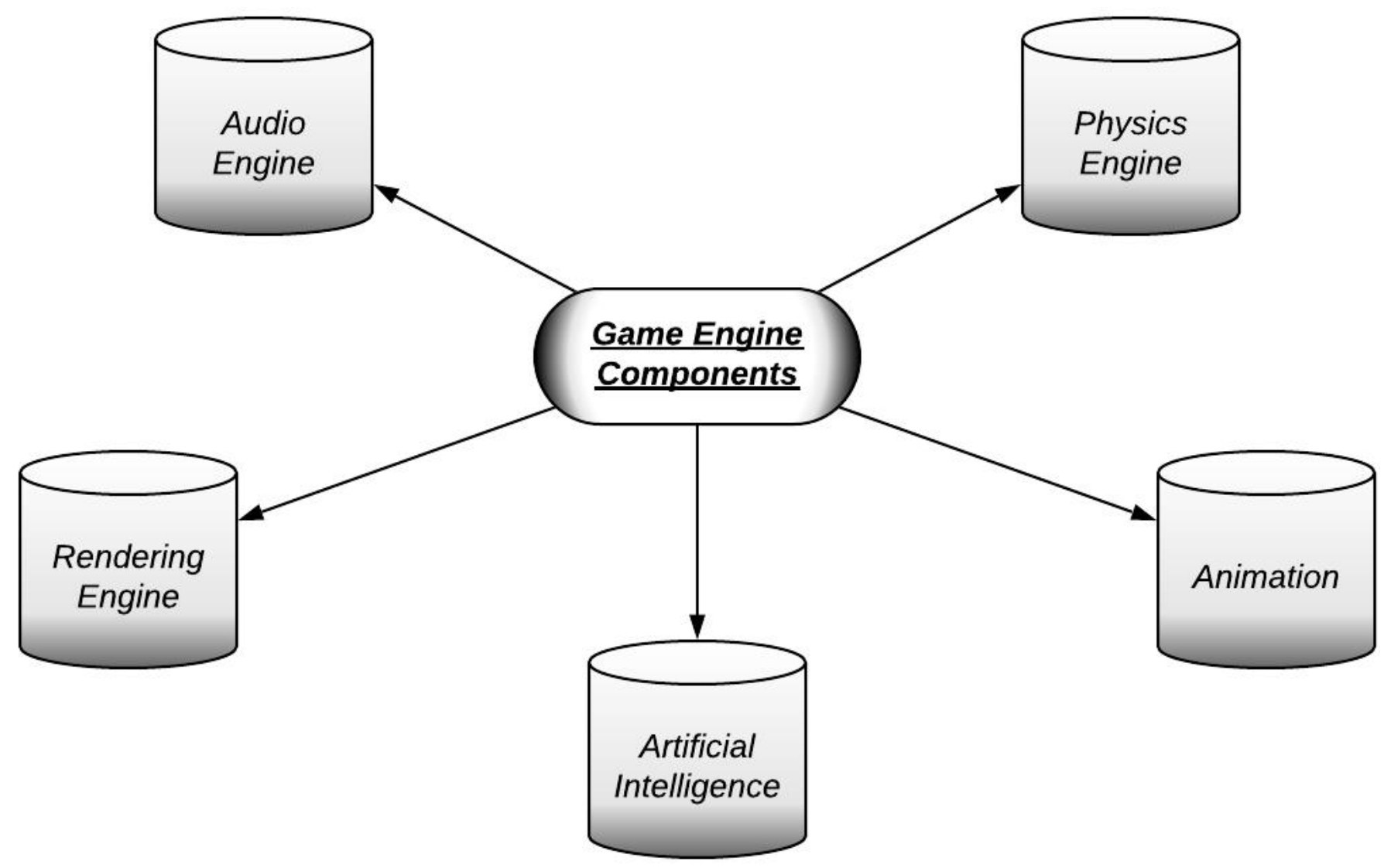

Game Engine Components Diagram

Figure 1. Game Engine Components Diagram

\section{Rendering Engine:}

The rendering engine works on creating 3D/2D animations by calling graphics APIs (Application Program Interface). This component/interface allows us to display objects by taking control of the GC (Graphic Card). (Šmíd A. 2017)

\section{Audio Engine:}

Sound design is an important part of each andevery game and having different sound effects and music play a huge role in the game's aura. (Šmíd A. 2017)

\section{Physics Engine:}

A computer software in game engines provides simulation to physical systems. For example, body dynamics (hard and soft body dynamics), fluid dynamics. (Šmíd A. 2017)

\section{Animation:}

Nowadays, animation is required in most of the games, animations are usually made inside the game engine itself. A character pose or a rotating ball are animated for example. (Šmíd A. 2017)

\section{Artificial Intelligence:}




\section{Journal of Student Research}

Fourth Middle East College Student Research Conference, Muscat, Sultanate of Oman

Moving the created player character around is of course being controlled by the developer. But what about the other objects around you? Basically, AI enables us to create a set of actions and gives it to the object. For example: Object [X] moves from point A to B without the user control but the $\mathrm{AI}^{\prime}$. This is a very crucial part in game development while using the engines. (Šmíd A. 2017; Christopoulou. E \& Xinogalos. S. 2017)

Based on the "Comparison Framework" that was made by Petridis et al. and Pattrasitidecha, in 2017, a detailed table was made to demonstrate the differences between both engines on the 5 major components of an engine:

Figure 2. Major Components Comparison 1

Figure 3. Major Components Comparison 2

Figure 4. Major Components Comparison 3

Figure 5. Major Components Comparison 4

\section{Results/Discussion (Table)}

In the table above, rendering engine had several sub categories. Textures: textures are the base of all game engines, and is divided into basic textures, multi-textures and procedural textures (As stated in Autodesk Knowledge Network, n.d.). Procedural textures are used to create different shapes for attributes, and they are mathematical functions that enable unlimited usage of resolution, (it can be either 2D or 3D). Lighting is very essential when it comes to light and make reflections (E.g. Sun lights). Per Vertex lighting, calculates lights on 3D models and then inserts and gathers all the results to get the lighting per pixels on the model's face. Lighting mapping is pre-computing the brightness/amount of light on a surface (Unity Manual, 2018). Gloss scales the roughness of the surface, either smooth or shiny (AWS, n.d.) Anisotropic reflections are basically like any other regular reflection, the only additional thing that it does is stretching and blurring (Blevins, N., 2014). The third important part in the rendering engine is the shadows, shadow mapping and volume are both alternatives to each other. Shadow mapping creates shadows out of an object if the created light sources do not hit that surface. Shadow volume is basically stretching out a mesh (net) of polygons from an object in a direction that would create the shadow (Williams, G., 2017). Within the rendering engine lies some special effects, environmental mapping, particle system and lens flare. Environmental mapping is a technique that is used to adjust the brightness of a reflected light. Particle system is all about simple meshes moving together that would create an effect (example: clouds) (Unity Manual, 2018). Lens flares are bright scattered light that produce noticeable effects if it is used correctly. In rendering engine, unreal engine takes the winner's flag since unity is missing 3 sub features which are: multi-textures, shadows volume and light mapping. Both engines are producing great results in the other major components. Clearly as the table states, both engines are well developed engines. However, there are other few important comparisons which are going to clarify the practicability and graphics quality of each engine. (Petridis et al. and Pattrasitidecha, 2017)

\section{Practicability}

During the research, the important functionalities with differences and similarities between both 


\section{Journal of Student Research}

Fourth Middle East College Student Research Conference, Muscat, Sultanate of Oman

engines were identified. Both Unreal Engine and Unity Engine provide a UI with the most necessary tools to develop a game. However, Unreal Engine's UI is more complicated than that of Unity. (Christopoulou. E \& Xinogalos. S., 2017)

One of the major differences between both engines is that the code language that is required to create a game on developer's own customization. Unity uses C\# code to create its script, it is necessary to customize the assets that you use or create your own (Petty, J. n.d). On the other hand, Unreal Engine uses $\mathrm{C}++$, an object-oriented language (Denham, T. n.d). Since Unreal engine is providing us with blueprints, coding using $\mathrm{C}++$ is not necessary unless the game developer wants to make his own customizations, but in the end, both do have assets which can help to develop the game. Moreover, Unity store provides more assets which will support beginners, but not unreal engine's store (Christopoulou. E \& Xinogalos. S. 2017)

It is worth mentioning that many tutorials regarding game development in Unity arefreely available, but very few in Unreal. This is a major difficulty faced by a a free learner.

\section{Graphics Quality}

In the previous years, the graphics quality gap was very huge between both engines, Unreal engine had the upper hand on that. However, with the improvement in technology, unity too managed to develop that part of it and till the gap and come very close to with almost no differences (Game designing, 2019). For an example (Unity Guruz.2019):

Figure 6. Graphics Quality (Unreal Engine right-side, Unity Engine left-side)

\section{Future Research Directions}

Learning generally about the top game engines and the way they process, shows us how big the gaming world is. The engine itself consists of several functions and features. This is why game development is better with a team to produce a better quality product. iIn near future, developing a game will be a good start and creating online content and tutorials for other people to understand and learn more about game engines, which will assist them to choose the game engine that they want to use.

\section{Conclusion}

All in all, both engines are pretty much similar, Unity engine is recommended if the aim is for an easier start, more beginner guides and more assets. Unreal engine on the other hand is very much recommended if thechallenge isthe aim and blueprints are going to make things easier, no learning codes are required. The table that was done by Petridis et al. and Pattrasitidecha, 2017, has shown that Unity Engine and Unreal Engines are rich with features and functionalities. As the graphical design made by Unity Guruz.2019 displays, the major gap in graphics between both engines is now very small, which means graphics are no issue these days on both engines. Using a game engine requires several skills and can be used for several uses. Unreal Engine is a good engine with better light effects than Unity engine. But Unity engine produces better shadows quality than Unreal engine. Both engines have their advantages and disadvantages, but it all depends on the skills and requirements that one has and what one wants to achieve with them.

\section{List of figures pg.}

1 Game Engine Components Diagram …................ 2 
1.2 Graphics Quality (Unreal Engine right-side, Unity Engine left-side)

\section{Acknowledgement:}

I would like to express my thanks to Mrs.Dhanalakshmi my research advisor for guiding me to complete my research. She encouraged and assisted me to pursue the path of game development and gave me the required support towards finishing this paper and to start my new journey to become a successful game developer.

\section{References:}

Game development. (n.d.). FreeCodeCamp Web site. Retrieved 16 November, from https://guide.freecodecamp.org/game-development/

Christopoulou. E \& Xinogalos. S (2017). Overview and comparative analysis of game engines for desktop and mobile devices. International Journal of Serious Games, 4(4), 21-36.

Pattrasitidecha, A. (2014). Comparison and evaluation of 3D mobile game engines, Master of Science Thesis in the Program International Design.

Petridis, P., Dunwell, I., Panzoli, D., Arnab, S., Protopsaltis, A., Hendrix, M. and Freitas, S., Game engines selection framework for high-fidelity serious applications, international journal of interactive worlds, Vol 2012(2012), 19 pages.

Šmíd A. (2017), Comparison of Unity and Unreal Engine.

Unreal engine vs unity: which is better? (2019). Game designing Web site. Retrieved 21 November, from https://www.gamedesigning.org/engines/unity-vs-unreal/

How do game engines work?. (2016). Interesting engineering Web site. Retrieved 21 November, from https://interestingengineering.com/how-game-engines-work

Concepts and feature of game engines. (2017). UKEssays Web site. Retrieved 23 November, from https://www.ukessays.com/essays/computer-science/concepts-features-game-engines-6438.php

5 years of unity vs. unreal: who is leading the war of giants. (2019). Programace Web site. Retrieved 25 November, from https://program-ace.com/blog/5-years-of-unity-vs-unreal/

Denham, T. What is unreal engine?. (n.d.). Concept art empire Web site. Retrieved 25 November, from https://conceptartempire.com/what-is-unreal-engine/

Petty, J. What is Unity 3D \& What is it used for?. (n.d.). Concept art empire Web site. Retrieved 25 November, from https://conceptartempire.com/what-is-unity/

Unity Guruz, (2019, April 10). Unity vs Unreal: Graphics Comparison 2019 (HDRP DEMO). File retrieved from https://www.youtube.com/watch?v=sMIi Z33pkw

Autodesk Knowledge Network. (n.d.). Procedural textures. Retrieved from https://knowledge.autodesk.com/support/maya/learn-explore/caas/CloudHelp/cloudhelp/2016/ENU/ Maya/files/GUID-B2C969C0-48CD-45AB-8C7B-E6FC9E34AD19-htm.html

AWS. (n.d.). Working with Gloss Maps. Retrieved from https://docs.aws.amazon.com/lumberyard/latest/userguide/mat-maps-gloss.html 


\section{Journal of Student Research}

Fourth Middle East College Student Research Conference, Muscat, Sultanate of Oman

Blevins, N. (2014). Anisotropic Reflections in The Real World. Retrieved from http://www.neilblevins.com/cg_education/aniso_ref_real_world/aniso_ref_real_world.htm

Unity Manual. (2018). Lightmapping: Getting started. Retrieved from https://docs.unity3d.com/Manual/Lightmapping.html

Williams, G. (2017). What is the difference between Shadow Volume and Shadow Mapping in Computer Graphics?. Retrieved from https://www.quora.com/What-is-the-difference-betweenShadow-Volume-and-Shadow-Mapping-in-Computer-Graphics 